\title{
Effect of a Single Shot Sciatic Nerve Block Combined with a Continuous Femoral Block on Pain Scores after Knee Arthroplasty. A Randomized Controlled Trial
}

\author{
Raul Carvalho $^{1,2}$, Luisa Calixto ${ }^{1}$, Jose Pedro Braganca ${ }^{1}$ \\ ${ }^{1}$ Serviço de Anestesia, Centro Hospitalar do Porto, Porto, Portugal; ${ }^{2}$ Departamento de Ciências da Informação e da Decisão em \\ Saúde, Faculdade de Medicina, Universidade do Porto, Porto, Portugal. \\ Email:raul@meo.pt
}

Received July $3^{\text {rd }}, 2012$; revised August $4^{\text {th }}, 2012$; accepted August $22^{\text {nd }}, 2012$

\begin{abstract}
Background and Purpose: Perioperative pain after total knee arthroplasty (TKA) is reported as severe in up to $60 \%$ of patients. Continuous femoral nerve blocks (CFNB) are a choice for major knee repair, but controversies remain about the need of supplemental sciatic nerve blocks (SNB) for better analgesia. Our aim is to assess the effect of the association of a SNB to a CFNB to reduce postoperative pain after TKA. Methods: A prospective randomized, single blinded, controlled study, on 50 patients undergoing TKA. Control group received a CFNB before general anesthesia; in the intervention group a single shot SNB was added after the CFNB was done. After the end of surgery all patients started a continuous local anesthetic infusion through the femoral catheter in the PACU (post-anesthesia care unit). Pain scores were measured in the PACU and at $12 \mathrm{~h}$ and $24 \mathrm{~h}$ postoperative using a visual analog scale (VAS). Results: VAS pain scores $(\mathrm{mm})$ were lower and statistically significant for the intervention group up to $12 \mathrm{~h}$ postoperative: PACU admission mean $\mathrm{VAS}=59.4$ vs $30.2, \mathrm{P}=0.001$; at $12 \mathrm{~h}$ mean $\mathrm{VASr}=26.1$ vs $9.2, \mathrm{P}=0.006$; at $24 \mathrm{~h}$ mean $\mathrm{VASr}=30.1$ vs 32.7, $\mathrm{P}=0.723$. Conclusions: The association of a single shot SNB with a CFNB significantly reduces postoperative pain scores after TKA up to $12 \mathrm{~h}$. At $24 \mathrm{~h}$ there are no differences between groups.
\end{abstract}

Keywords: Postoperative Pain; Knee Arthroplasty; Regional Anesthesia; Nerve Blocks

\section{Introduction}

Postoperative pain is a concern for patients submitted to knee arthroplasty and it has been reported as being moderate in up to $30 \%$ and severe in $60 \%$ of the patients [1]. There is an ongoing debate on which type of anesthesia is better for these patients but there is no clear evidence of any advantage of regional versus general anesthesia on long-term outcomes such as morbidity, mortality or rehabilitation [2]. Regional anesthesia provides better postoperative analgesia, and may improve early rehabilitation with fewer side effects when compared to intravenous opiates $[2,3]$, but there are no clear conclusions on what type of regional anesthesia is better.

Peripheral nerve blocks have less side effects compared to neuraxial blocks and femoral nerve blocks are well established as an option for postoperative analgesia in knee replacement surgery [4] and is now recommended by the Procedure Specific Postoperative Pain Management Working Group [5]. There is uncertainty on whether sciatic or obturator nerve blocks are needed as adjuncts to a femoral block to improve postoperative analgesia, and conflicting results have been published [6-11]. A review by Hogan et al. states the benefits of peripheral nerve blocks in analgesia for hip and knee analgesia, but fails to recognize which are the most effective blocks [12]. Two meta-analysis recently published showed inconclusive evidence to define the effect of adding sciatic nerve block (SNB) to femoral nerve block (FNB) on acute pain and related outcomes due to the lack of good randomized studies comparing both techniques [13]. An editorial by Ilfeld \& Madison in 2011 recognized the lack of consensus and the need for additional research [14].

The aim of this study is to determine if a combination of a single shot SNB and continuous femoral nerve block (CFNB) provide better postoperative analgesia when compared to isolated CFNB in patients undergoing total knee arthroplasty (TKA).

Our hypothesis is that the association of a single shot SNB to a CFNB can reduce mean pain scores at least $15 \%$ when compared to CFNB alone, as we considered this to be a clinical significant difference. 


\section{Material and Methods}

The study protocol was approved by the Ethics Committee of Centro Hospitalar do Porto and received institutional approval. It was registered in Clinicaltrials.gov under the identifier NCT01337115.

\subsection{Type of Study}

Interventional, prospective, randomized, controlled, parallel group, single blinded, treatment efficacy study.

\subsection{Selection of Participants}

Eligible participants were drawn from 167 consecutive patients undergoing unilateral TKA from April 2011February 2012, in the Orthopedic Department of Centro Hospitalar do Porto, Portugal.

Patients had to be anesthetized by one of 5 senior anesthesiologists of the orthopedic anesthesia group of the department. Exclusion criteria were refusal to give informed consent, contraindication to general anesthesia, infection at needle insertion site, coagulation disorders, preexisting neurologic disorders, known allergies to local anesthetics, diclofenac or tramadol, severe dyspepsia, ASA status 4 or 5 , weigh less than $50 \mathrm{~kg}$, body mass index $>40$, inability to understand and use the visual analogue scale (VAS) pain score.

Group allocation was made by a computer generated random number list (Office Excel 2011 ${ }^{\circledR}$, Microsoft Corporation, Redmond, WA, USA) and codes stored in opaque sealed envelops.

Written consent was obtained immediately prior to envelope opening and group allocation.

\subsection{Intervention}

We used the Stimuplex ${ }^{\circledR}$ HNS12 nerve stimulator (B. Braun Melsungen AG, Germany) to perform the nerve blocks. For CFNB, the paravascular approach [15] was used to identify the femoral nerve. We considered a positive location when quadriceps contraction (patellar elevation) was elicited with a current of $0.46 \mathrm{~mA}$ or less [16]. Ten milliliters of ropivacaine $0.375 \%$ were injected through the needle (Contiplex ${ }^{\circledR}$ Tuohy $18 \mathrm{G} 50 \mathrm{~mm}$ length needle, with $20 \mathrm{G}, 100 \mathrm{~mm}$ catheter, B. Braun Melsungen AG, Germany), a catheter was then inserted and $20 \mathrm{ml}$ of ropivacaine $0.375 \%$ were administered through the catheter.

For the single shot sciatic block, we used the anterior approach described by Chelly [17]. A Stimuplex ${ }^{\circledR}$ insulated needle $21 \mathrm{G} \times 100 \mathrm{~mm}$ or $20 \mathrm{G} \times 150 \mathrm{~mm}$ was used (B. Braun Melsungen, Germany) and $25 \mathrm{ml}$ of ropivacaine $0.375 \%$ were administered when either the common peroneal or tibial nerve (dorsiflexion or plantar flexion of the foot) were identified with a stimulation of
$0.46 \mathrm{~mA}$ or less [16]. Blocks success was assessed by absence of thermal sensitivity with an alcohol swab on the anterior region of the thigh and dorsum of the foot.

All patients were then induced to general anesthesia with propofol $(1.2-2 \mathrm{mg} / \mathrm{kg})$ and fentanyl $(0.15-0.2$ $\mu \mathrm{g} / \mathrm{kg}$ ) and maintained with short action halogenated anesthetics (Desflurane or Sevoflurane). Airway was maintained with laryngeal mask airway and pressure support ventilation. Additional fentanyl was given during the procedure if attending anesthesiologist considered necessary. Total intraoperative fentanyl dose was registered.

Thirty minutes before the end of the procedure, IV ketorolac $30 \mathrm{mg}$ and paracetamol $1000 \mathrm{mg}$ was administered.

On arrival to the post-anesthesia unit (PACU), neurologic function was assessed by an independent observer and an infusion of ropivacaine $0.2 \%$ was started on the femoral catheter at a rate of $8 \mathrm{ml} / \mathrm{h}$ using an Easypump $^{\circledR}$ C-bloc RA 400-8 (B. Braun Melsungen AG, Germany).

Both groups got diclofenac $50 \mathrm{mg}$ q12h $\mathrm{PO}$ and paracetamol $1000 \mathrm{mg}$ q6h PO and as rescue analgesia, tramadol $100 \mathrm{mg}$ q6h prn IV. Thromboprophylaxis with LMWH was started in the first $24 \mathrm{~h}$ and maintained for 5 weeks.

\subsection{Data Collection}

We assessed pain using a standard $100 \mathrm{~mm}$ VAS (VAS = 0 no pain; $\mathrm{VAS}=100$ worst pain).

At 15 - 30 minutes after arrival to PACU, with the patient awake and fully collaborating, there was a first pain evaluation. If VAS $>30$, and the patient asked for treatment, morphine $2 \mathrm{mg}$ IV was given every 15 minutes until VAS 30 or less and patient was comfortable.

VAS pain scores, morphine consumption, and time of discharge from PACU were recorded. Presence of toe movements was confirmed before PACU discharge.

Additional measurements of VAS pain scores (VASr: at rest; VASm: with movement) at $12 \mathrm{~h}$ and $24 \mathrm{~h}(+/-2 \mathrm{~h})$ after surgery were made.

Rescue tramadol usage and complications (falls, foot paralysis, paresthesias, nausea and vomiting) were registered up to $24 \mathrm{~h}$.

One month after surgery, subjects were contacted by telephone and asked to rate their satisfaction with postoperative analgesia in a categorical scale of 4 groups (bad; average; good; excellent).

Investigators blinded for the allocation group of the subjects made all assessments.

\subsection{Variables}

Pain scores on VAS is a continuous variable with a range of $0-100$. 
The primary outcome of the trial is the difference in mean scores from VAS pain assessment in both groups $(0=$ no pain; $100 \mathrm{~mm}=$ worst pain $)$ at three predefined moments on the first $24 \mathrm{~h}$ after surgery: $15-30 \mathrm{~min}$ (in PACU); $12 \mathrm{~h}$ and $24 \mathrm{~h}(+/-2 \mathrm{~h})$.

Tramadol consumption and patient satisfaction are ordinal variables.

Side effects and complications (as previously defined) are reported and incidence calculated.

\subsection{Statistical Analysis}

Fifty subjects were randomized into Control $(\mathrm{CFNB} ; \mathrm{n}=$ $25)$ and Intervention $(\mathrm{CFNB}+\mathrm{SNB} ; \mathrm{n}=25)$ groups.

Power analysis for sample size was calculated using a web free application [18] and was designed to detect minimum differences of $15 \%$, with type I error 0.05 and $80 \%$ power.

A two-tailed t-test for independent variables was used to analyze differences in mean VAS scores in intentionto-treat manner.

The normal distribution of the continuous variables was assessed by the Kolmogorov-Smirnov test.

Our null hypothesis states that there is no difference between the mean pain scores of control and intervention groups. Statistical significance is assumed if $\mathrm{P}<0.05$.

Ordinal variables were analyzed with Fisher's exact test [19].

Continuous variables analysis was done with $\mathrm{IBM}^{\circledR}$ PASWSatistics 18.0.3 for MAC (IBM Corporation, Somers, NY, USA).

\section{Results}

A total of 167 subjects were assessed for eligibility from April 2011 until February 2012. A high number of excluded patients were due to the fact that only 5 senior anesthesiologists were involved in the study.

Figure 1 shows a flowchart of patient selection and participation in the study.

Two patients were excluded before the $12 \mathrm{~h}$ assessment because of femoral catheter displacement. The drop out of 2 patients in the control group (CFNB) did not affect the statistical power because the sample needed only 23 patients in each group.

Table 1 shows the patient demographic data. There were no differences between groups. The higher number of female subjects is consistent with the reported higher risk of osteoarthritis in women and with the average caseload of our department.

Table 2 shows the overall results. Pain scores are significantly reduced at PACU admission $(\mathrm{P}=0.001)$ and at 12 hours post-operative $(\mathrm{P}=0.006)$ on the intervention group (CFNB $+\mathrm{SNB})$. These differences remained if pain was assessed for movement. Figure 2 shows a graph bar with $95 \% \mathrm{CI}$ for the main results.

Morphine consumption $(\mathrm{P}<0.001)$ and time spent in PACU $(\mathrm{P}=0.045)$ are also reduced in the intervention group. PACU time is influenced by several organizational factors that were not controlled and should be valued with care.

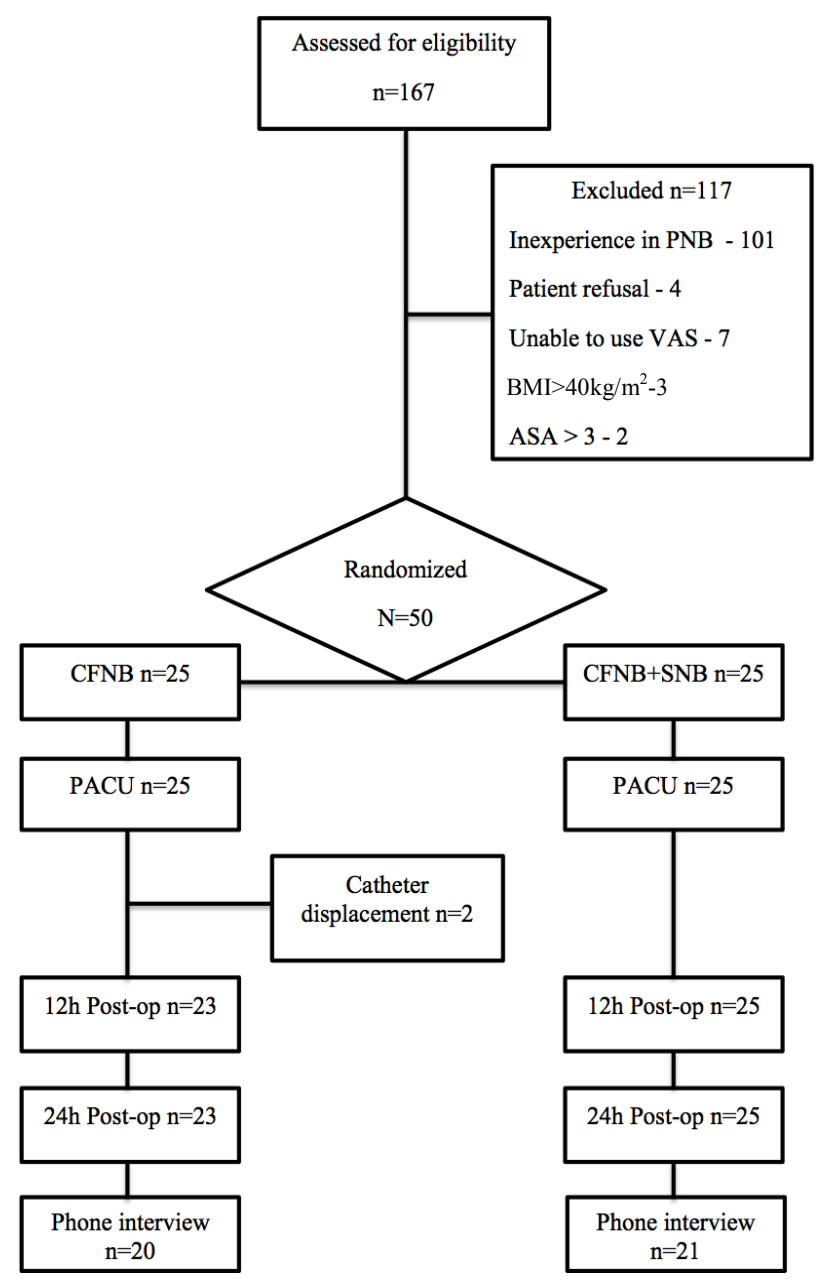

Figure 1. Participants flowchart.

Table 1. Participants characteristics.

\begin{tabular}{lcc}
\hline & $\begin{array}{c}\text { CFNB } \\
(\mathrm{n}=25)\end{array}$ & $\begin{array}{c}\text { CFNB }+ \text { SNB } \\
(\mathrm{n}=25)\end{array}$ \\
\hline Sex (female, male) & 20,5 & 16,9 \\
Age, mean (SD), y & $68(10)$ & $65(9)$ \\
ASA physical status I, II, III & $0,22,3$ & $3,21,1$ \\
Body mass index (SD), $\mathrm{kg} / \mathrm{m}^{2}$ & $28.1(4.6)$ & $29.9(3.7)$ \\
\hline
\end{tabular}

Continuous variables are reported as mean (SD); Categorical variables are reported as absolute numbers. 
Table 2. Global results: pain scores, PACU time and morphine consumption.

\begin{tabular}{|c|c|c|c|c|}
\hline & $\operatorname{CFNB}(\mathrm{n}=25)$ & $\mathrm{CFNB}+\mathrm{SNB}(\mathrm{n}=25)$ & $95 \% \mathrm{CI}$ & $\mathrm{P}$ \\
\hline Intraoperative fentanyl $(\mu \mathrm{g})^{*}$ & $208.0(64.0)$ & $172.0(66.3)$ & {$[(-1.06)-73.06]$} & 0.057 \\
\hline Pain PACU admission VAS ${ }^{*}$ & $59.4(27.2)$ & $30.2(30.5)$ & {$[12.83-45.65]$} & 0.001 \\
\hline Max PACU pain VAS* & $69.8(24.0)$ & $36.7(31.4)$ & {$[17.23-49.01]$} & $<0.001$ \\
\hline Morphine PACU $(\mathrm{mg})^{*}$ & $6.2(3.5)$ & $2.2(2.7)$ & {$[0.89-2.18]$} & $<0.001$ \\
\hline PACU time $(\mathrm{min})^{*}$ & $126.5(47.3)$ & $97.2(53.2)$ & {$[0.65-57.91]$} & 0.045 \\
\hline Pain 12 h VASr, ${ }^{* * *}$ & $26.1(23.8)$ & $9.2(14.8)$ & {$[5.52-28.33]$} & 0.006 \\
\hline Pain 12 h VAS m, & $36.2(26.6)$ & $18.6(22.9)$ & {$[3.23-32.00]$} & 0.018 \\
\hline Pain 24 h VASr ${ }^{* * *}$ & $30.1(25.0)$ & $32.7(24.5)$ & {$[(-16.93)-11.83]$} & 0.723 \\
\hline Pain 24 h VASm ${ }^{* * *}$ & $49.8(29.7)$ & $46.9(29.0)$ & {$[(-14.12)-20.00]$} & 0.730 \\
\hline
\end{tabular}

CFNB — Continuous femoral nerve block; SNB — Sciatic nerve block; VASr—VAS at rest; VASm—VAS with movement; ${ }^{*}$ - mean $(\mathrm{SD}) ;{ }^{* *}$ - CFNB n= 23.

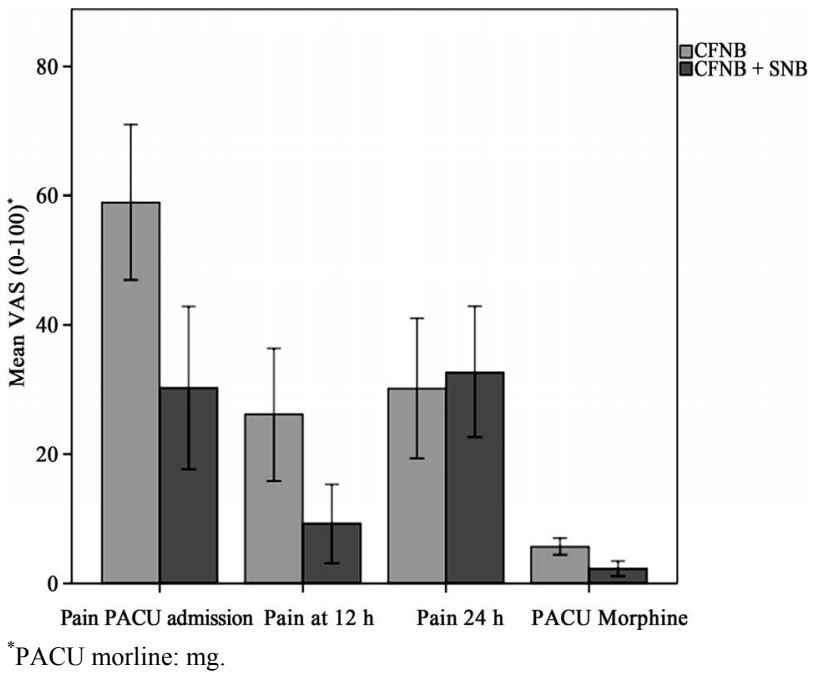

Figure 2. CI (95\%) for main results.

Only 19 patients used tramadol as rescue analgesia $(\mathrm{CFNB} n=9$; CFNB + SNB $n=10)$ and there are no differences between groups (Fisher's exact test $\mathrm{P}=0.459$ ). No serious complications were found in either group. Two patients had mild nausea after tramadol administration.

At $24 \mathrm{~h}$ none of the subjects of either group had motor block or paresthesias in the sciatic territory. Weakness of quadriceps femoral muscle was present. Femoral catheter stayed in place up to $48 \mathrm{~h}$ and was managed by the Acute Pain Unit as normally with controlled ambulation starting at $48 \mathrm{~h}$ postoperative. In spite of three attempts, 9 patients could not be contacted for the final satisfaction assessment. Fisher's exact test showed no difference in overall satisfaction with analgesia $(\mathrm{P}=0.537)$.

\section{Discussion}

A SNB reduced postoperative pain for up to 12 hours after TKA. This reduction is seen at rest and with movement and is from intense to moderate pain. At 24 hours postoperative no effect can be seen.

Morphine consumption is also reduced in the intervention group in PACU. Using analgesic consumption for measuring pain has limitations [20], however these results are in accordance with the measurements from VAS and support the benefits of associating sciatic and femoral blocks for TKA. Intraoperative analgesic techniques may influence early postoperative pain scores. Using a general anesthesia with short acting inhalational agents (sevoflurane or desflurane) minimized the possibility of this bias. The intraoperative fentanyl used by both groups was not statistically different so its influence in early postoperative pain scores would be minimal.

The resolution of the SNB may explain the absence of differences in pain scores at $24 \mathrm{~h}$, but to confirm this would need a different study design as the use of a single shot SNB limits our capability to detect effects only on the early postoperative period.

Contradicting results from several studies [8] and studies comparing the efficacy of different techniques [21] led to the absence of strong evidence to recommend or not the SNB for analgesia after TKA [13]. We directly compare the two techniques (CFNB vs CFNB + SNB) and show an effect of the SNB. Limiting our study to the first $24 \mathrm{~h}$ postoperative and directly measuring pain scores as a primary outcome instead of proxy measurements allowed for less confounding results.

Nineteen patients $(\mathrm{CFNB}=9 ; \mathrm{CFNB}+\mathrm{SNB}=10)$ used rescue analgesia (tramadol $100 \mathrm{mg} \mathrm{6/6} \mathrm{h} \mathrm{prn} \mathrm{IV)}$ 
with no difference between groups (Fisher's exact test $\mathrm{P}$ $=0.459$ ). Patients in the intervention group needed analgesia only after $12 \mathrm{~h}$ post-operative and the control group used it from the immediate post-operative period. The onset of new pain from the resolution of the SNB in patients of the CFNB + SNB group may be an explanation for this difference. The control group also had a wider range of the tramadol dose $(0-300 \mathrm{mg})$ vs the intervention group $(0-200 \mathrm{mg})$. Due to the small number we cannot give statistical significance to these findings and this is a limitation of our study that was not powered to evaluate these findings.

The overall patient satisfaction with analgesia was very high in both groups (Good/Very Good-Control $85 \%$; Intervention $86 \%$ of the valid subjects; Fisher's exact test $\mathrm{P}=0.537)$, but there were $18 \%$ of patients that did not answer our phone interview (5 in the CFNB vs 4 in the CFNB + SNB group) and the study is underpowered for this variable.

Patients had difficulties in distinguishing between anesthesia/analgesia experience from the overall hospital experience and this may be an important bias in this parameter. Satisfaction assessment probably should have been done earlier to be more objective. A study about patient experiences regarding the use of regional anesthesia for hip and knee arthroplasty shows the difficulties and complexity in assessing patient preferences and establishes the surgeons and anesthetists preferences as the most influential parameter on patient preferences [22].

An important limitation of our study is that it is single blinded, and the patient is aware if a SNB was performed or not and could influence patients complaints, but due to the risk of complications we chose not to perform sham SNBs.

We do not assess the importance of peripheral nerve blocks in rehabilitation [2], nor the effect of the femoral continuous block beyond the first $24 \mathrm{~h}$, but the short term efficacy and safety can be seen from the low pain scores and absence of complications. A recent study showed no differences in time-to-discharge readiness or flexion rate at time of discharge irrespective of the use of a single injection or continuous SNB [23].

\section{Conclusions}

The association of a single shot sciatic nerve block to a continuous femoral nerve block significantly reduces pain scores after knee arthroplasty up to $12 \mathrm{~h}$ postoperative.

This association also reduces PACU time stay and morphine consumption.

\section{REFERENCES}

[1] J. Bonica, "Postoperative Pain," In: J. Bonica, Ed., The
Management of Pain, Lea \& Febiger, Philadelphia, 1990, pp. 461-480.

[2] A. J. Macfarlane, G. A. Prasad, V. W. Chan and R. Brull, "Does Regional Anesthesia Improve Outcome after Total Knee Arthroplasty?" Clinical Orthopaedics and Related Research, Vol. 467, No. 9, 2009, pp. 2379-2402. doi:10.1007/s11999-008-0666-9

[3] F. J. Singelyn, M. Deyaert, D. Joris, E. Pendeville and J. M. Gouverneur, "Effects of Intravenous Patient-Controlled Analgesia with Morphine, Continuous Epidural Analgesia, and Continuous Three-in-One Block on PostOperative Pain and Knee Rehabilitation after Unilateral Total Knee Arthroplasty," Anesthesia \& Analgesia, Vol. 87, No. 1, 1998, pp. 88-92.

[4] A. P. Boezaart, "Perineural Infusion of Local Anesthetics," Anesthesiology, Vol. 104, 2006, pp. 872-880. doi:10.1097/00000542-200604000-00033

[5] Prospect Working Group, "Procedure Specific Postoperative Pain Management," 2010.

http://www.postoppain.org

[6] S. J. Fowler, J. Symons, S. Sabato and P. S. Myles, "Epidural Analgesia Compared with Peripheral Nerve Blockade after Major Knee Surgery: A Systematic Review and Meta-Analysis of Randomized Trials," British Journal of Anaesthesia, Vol. 100, No. 2, 2008, pp. 154-164. doi:10.1093/bja/aem373

[7] J. R. Hebl, S. L. Kopp, M. H. Ali, T. T. Horlocker, J. A. Dilger, R. L. Lennon, B. A. Williams, A. D. Hanssen and M. W. Pagnano, "A Comprehensive Anesthesia Protocol that Emphasizes Peripheral Nerve Blockade for Total Knee and Total Hip Arthroplasty," The Journal of Bone \& Joint Surgery of America, Vol. 87, No. 2, 2005, pp. 6370. doi:10.2106/JBJS.E.00491

[8] H. W. Allen, S. S. Liu, P. D. Ware, C. S. Nairn and B. D. Owens, "Peripheral Nerve Blocks Improve Analgesia after Total Knee Replacement Surgery," Anesthesia \& Analgesia, Vol. 87, No. 1, 1998, pp. 93-97.

[9] B. Ben-David, K. Schmalenberger and J. E. Chelly, “Analgesia after Total Knee Arthroplasty: Is Continuous Sciatic Blockade Needed in Addition to Continuous Femoral Blockade?" Anesthesia \& Analgesia, Vol. 98, No. 3, 2004, pp. 747-749. doi:10.1213/01.ANE.0000096186.89230.56

[10] A. M. Morin, C. D. Kratz, L. H. Eberhart, G. Dinges, E. Heider, N. Schwarz, G. Eisenhardt, G. Geldner and H. Wulf, "Postoperative Analgesia and Functional Recovery after Total-Knee Replacement: Comparison of a Continuous Posterior Lumbar Plexus (Psoas Compartment) Block, a Continuous Femoral Nerve Block, and the Combination of a Continuous Femoral and Sciatic Nerve Block," Regional Anesthesia and Pain Medicine, Vol. 30, No. 5, 2005, pp. 434-445. doi:10.1016/j.rapm.2005.05.006

[11] C. P. Dang, E. Gautheron, J. Guilley, M. Fernandez, D. Waast, C. Volteau, J. M. Nguyen and M. Pinaud, "The Value of Adding Sciatic Block to Continuous Peripheral Nerve Blockade for Total Knee and Total Hip Replacement," Regional Anesthesia and Pain Medicine, Vol. 30, 2005, pp. 128-133.

doi:10.1097/00115550-200503000-00003 
[12] M. V. Hogan, R. E. Grant and L. J. Lee. "Analgesia for Total Hip and Knee Arthroplasty: A Review of Lumbar Plexus, Femoral, and Sciatic Nerve Blocks," The American Journal of Orthopedics, Vol. 38, No. 8, 2009, pp. E129-E133.

[13] J. E. Paul, A. Arya, L. Hurlburt, J. Cheng, L. Thabane, A. Tidy and Y. Murthy, "Femoral Nerve Block Improves Analgesia Outcomes after Total Knee Arthroplasty: A Meta-Analysis of Randomized Controlled Trials," Anesthesiology, Vol. 113, No. 5, 2010, pp. 1144-1162. doi:10.1097/ALN.0b013e3181f4b18

[14] B. M. Ilfeld and S. J. Madison, "The Sciatic Nerve and Knee Arthroplasty: To Block, or Not to Block-That Is the Question," Regional Anesthesia and Pain Medicine, Vol. 36, No. 5, 2011, pp. 421-423.

[15] A. P. Winnie, S. Ramamurthy and Z. Durrani, "The Inguinal Paravascular Technic of Lumbar Plexus Anesthesia: The '3-In-1 Block'," Anesthesia \& Analgesia, Vol. 52, No. 6, 1973, pp. 989-996.

[16] B. C. H. Tsui, "Electrical Nerve Stimulation,” In: B. C. H. Tsui, Ed., Atlas of Ultrasound and Nerve StimulationGuided Regional Anesthesia, Springer Verlag, New York, 2007, pp. 9-18.

[17] J. E. Chelly and L. Delaunay, "A New Anterior Approach to the Sciatic Nerve Block," Anesthesiology, Vol. 91, No. 6, 1999, pp. 1655-1660. doi:10.1097/00000542-199912000-00017

[18] R. V. Lenth, "Java Applets for Power and Sample Size,"
Journal of Animal Science, Vol. 85, No. 13, 2007, pp. E24-E29. http://www.stat.uiowa.edu/ rlenth/Power

[19] S. A. Joosse, "Fisher's Exact Test," 2011. http://www.in-silico.net/statistics/fisher_exact_test

[20] R. A. Moore, R. J. Mhuircheartaigh, S. Derry and H. J. McQuay, "Mean Analgesic Consumption Is Inappropriate for Testing Analgesic Efficacy in Post-Operative Pain: Analysis and Alternative Suggestion," European Journal of Anaesthesiology, Vol. 28, No. 6, 2011, pp. 427-432. doi:10.1097/EJA.0b013e328343c569

[21] A. F. Davies, E. P. Segar, J. Murdoch, D. E. Wright and I. H. Wilson, "Epidural Infusion or Combined Femoral and Sciatic Nerve Blocks as Perioperative Analgesia for Knee Arthroplasty," British Journal of Anaesthesia, Vol. 93, 2004, pp. 368-374. doi:10.1093/bja/aeh224

[22] F. Webster, S. Bremner and C. J. McCartney, "Patient Experiences as Knowledge for the Evidence Base: A Qualitative Approach to Understanding Patient Experiences Regarding the Use of Regional Anesthesia for Hip and Knee Arthroplasty," Regional Anesthesia and Pain Medicine, Vol. 36, No. 5, 2011, pp. 461-465.

[23] J. T. Wegener, B. van Ooij, C. N. van Dijk, M. W. Hollmann, B. Preckel and M. F. Stevens, "Value of SingleInjection or Continuous Sciatic Nerve Block in Addition to a Continuous Femoral Nerve Block in Patients Undergoing Total Knee Arthroplasty: A Prospective, Randomized, Controlled Trial," Regional Anesthesia and Pain Medicine, Vol. 36, No. 5, 2011, pp. 481-488. 